\title{
Implementation of a Drive-Through COVID-19 Mass Vaccination Site: Experiences from LouVax-Broadbent in Louisville, Kentucky
}

\author{
Ruth Carrico 1,2,5, PhD DNP FNP-C CIC FAAN; Sarah Beth Hartlage ${ }^{3,4}$, MD; Valenchia Brown ${ }^{1,2,5}$, MSN APRN FNP-C; Sarah Bishop ${ }^{1}$, MSN \\ APRN CCNS CIC; Luanne Didelot ${ }^{1}$, MSN; William Hayden ${ }^{5}$, BSN; Beverly Williams Coleman ${ }^{5,6}$, APRN; Sarah Tan ${ }^{1}$, BS; Delanor Manson ${ }^{5}$, \\ MA, BSN; Kellie Kane ${ }^{3}$, MD; Dawn Balcom ${ }^{1,5}$, DNP APRN FNP-C; Paul Kern ${ }^{3}$, MS-SSEM, MBA
}

${ }^{1}$ Division of Infectious Diseases, Center for Education and Training in Infection Prevention (CETIP), University of Louisville School of Medicine; ${ }^{2}$ Norton Infectious Diseases Institute, Norton Healthcare, Louisville Kentucky; ${ }^{3}$ Louisville Metro Public Health and Wellness (LMPHW); ${ }^{4}$ School of Public Health and Information Sciences (SPHIS), University of Louisville; ${ }^{5}$ Kentucky Nurses Association (KNA); ${ }^{6}$ University of Louisville School of Nursing

*ruth.carrico@nortonhealthcare.org

Recommended Citation: Carrico R, Hartlage SB, Brown V, et al. Implementation of a drive-through COVID-19 mass vaccination site: Experiences from LouVax-Broadbent in Louisville, Kentucky. Univ Louisville J Respir Infect 2022; 6(1):Article 3. doi: 10.18297/jri/vol6/iss1/3.

\begin{abstract}
Background: Response to the COVID-19 pandemic has required innovative approaches to vaccination, including methods to rapidly and safely immunize communities. A major challenge to such a response involved access to untapped healthcare worker resources. The Louisville, Kentucky, response involved the use of a large volunteer workforce to supplement local public health employees in planning and implementing a large-scale drive-through vaccination event. The objective of this manuscript was to outline processes involved in training a mass immunization workforce and implementating a large-scale COVID-19 community vaccination.
\end{abstract}

Approach: Competency-based and standardized training was provided for every volunteer before working their shift. Volunteers worked four-hour shifts, and training sessions were held at the beginning of every shift. Training included three overarching goals with associated procedures: to protect the vaccine supply, protect the individuals being vaccinated, and protect the healthcare workforce during a 17-week large-scale community drive-through vaccination campaign.

Results: Between January 4, 2021, and April 28, 2021, LouVax-Broadbent was open for operation for 82 days. A total of 109,529 doses of COVID-19 vaccine were administered at LouVax-Broadbent; 2,561 volunteers served the community at LouVax-Broadbent, yielding approximately 81,200 volunteer hours. Three needlestick injuries were reported, all of which occurred during safety device activation. Procedures that served to protect the vaccine resulted in a waste of 132 $(0.12 \%)$ of the 109,529 prepared doses.

Conclusions: Vaccination rate outcomes at LouVaxBroadbent demonstrated an ability to engage, train, and safely implement a large and dedicated group of volunteer healthcare and non-healthcare workers as part of a largescale community mass COVID-19 drive-through vaccination event.

\section{Background}

Novel approaches to immunization have been implemented in Louisville, Kentucky, for close to thirty years, with the first drive-through influenza vaccination occurring in 1994.[1] Provision of influenza immunization via drive-through became an annual event for the next ten years as the general public and healthcare providers grew to be more comfortable with vaccination occurring outside the traditional office setting. Through these experiences, Louisville, a moderately sized community of just under one million, demonstrated an ability to develop and implement innovative approaches to healthcare delivery. Interest in the drive-through process for immunization prompted the compilation of the activities into a book that was subsequently shared across the US and internationally.[2] Building upon provider and public acceptance, procedures for drive-through immunization as part of largescale public health response were developed, then used in 2009 in Louisville to address the H1N1 influenza pandemic. The 2009 mass H1N1 immunization event was held at the University of Louisville football stadium parking lot. Planning for the event was a collaboration between researchers, clinicians, and emergency management at the University of Louisville and the full spectrum of planners at the Louisville Metro Department of Public Health \& Wellness (LMPHW) and 
the City of Louisville. As a result of those efforts, approximately 20,000 H1N1 influenza vaccine doses were administered to community members in less than 18 hours as part of the vaccine roll-out. National and international media coverage chronicled the event as the largest mass immunization drive-through effort to have occurred anywhere in the world.[3] To address national and international interest in drive-through vaccination, a new 'how-to' manual was published in 2020 as concerns about an emerging infection pandemic became a reality.[4]

Interventions to address the current COVID-19 pandemic have resulted in renewed interest in how rapid large-scale vaccination could be implemented in local communities. Making vaccine available to entire communities during the COVID-19 pandemic required that a respiratory protection component be added to the standard safe immunization practices, resulting in a broader approach to infection control.[5] Public health leadership in Louisville, Kentucky, embraced the notion of drive-through mass immunization as the most effective initial approach for administering COVID-19 vaccine to the general public as part of pandemic response. Release of three COVID-19 vaccines through emergency use authorization (EUA) brought a new level of complexity to mass immunization but further underscored the urgent need for training as a foundation for community-based rapid mass immunization capabilities.[6-8]

The objective of this paper is to outline the processes involved in training a mass immunization workforce and implementing a large-scale COVID-19 community vaccination effort in Louisville, Kentucky.

\section{Approach}

Planning for COVID-19 mass vaccination using a drivethrough approach began in late summer 2020. Operational documents from the $2009 \mathrm{H} 1 \mathrm{~N} 1$ influenza mass vaccination event were evaluated by public health planners to determine their utility and applicability as a planning guide. Broadbent Arena, a large indoor area located on state property, was identified as a centrally located and ideal location where indoor vaccination could occur and be minimally impacted by weather. Broadbent Arena had been regularly used for monster truck shows, and the ventilation system was capable of addressing the environmental concerns of vehicular exhaust in the enclosed arena. Fully opening entry and exit arena doorways provided additional airflow. Air quality and ventilation capabilities were evaluated and deemed safe and acceptable by Louisville Metro Public Health and Wellness (LMPHW) environmentalists, both at the time of planning and daily throughout the vaccination event. The Louisville drive-through mass vaccination site was aptly named LouVax-Broadbent.
The size and configuration of the arena enabled planning for three zones of operation. Zone 1 was the area outside the arena entrance where cars were organized into lines in preparation for entry into the arena. Uniformed police officers were also stationed in Zone 1 to assist with traffic control, if needed, and provide visible security. A pre-registration process aligned with the federal government's phased approach for vaccination was used to organize population groups sent to LouVax-Broadbent. These population groups included healthcare workers, teachers, first responders, and older adults. Registration was managed through a LMPHW call center. Individuals who had been preregistered were provided with an auto-response email containing a QR code, and they were advised to have that QR code accessible upon arrival at the arena. The QR code was shown to Zone 1 personnel; then, the individual was guided toward Zone 2. Electronic access to registration lists was available for Zone 1 personnel so that they could assist those who had lost or were unable to produce their individual QR code.

Cars entered Zone 2 through a large cargo door, where they were immediately directed by trained personnel into one of six vaccination lanes. Although the process was designed to operate via drive-through, there was also a designated area in Zone 2 where walk-up traffic could be accommodated. Zone $2 \mathrm{~A}$ provided a place for bus transportation, shared ride transportation (e.g., Uber, Lyft), groups requiring interpreters, and other special needs unable to be addressed efficiently via drive-through. Once vaccine was administered, cars would exit the arena and be directed toward Zone 3 . Zone 3 was an area outside the arena where postvaccination monitoring occurred. Figure 1 shows the schematics for LouVax-Broadbent, and Figure 2 shows an aerial view of the arena and adjacent areas. Training for volunteers working in Zones 1 and 3 was provided by LMPHW and the City of Louisville. Training for Zone 2 was provided by personnel from the University of Louisville Division of Infectious Diseases Center for Education and Training in Infection Prevention (CETIP).

Training the workforce for implementation of mass vaccination at LouVax-Broadbent involved three overarching objectives: 1 ) protect the vaccine, 2) protect the person receiving the vaccine (recipient), and 3 ) protect the workforce involved in vaccine administration.

\section{LouVax-Broadbent workforce}

Following a two-week pilot phase, full operation began on January 4, 2021, and continued for 17 weeks, with the last day of operation on April 29, 2021. A unique aspect of this COVID-19 mass vaccination event was its use of volunteers for the vast majority of the workforce in all three Zones. Volunteer healthcare workers were recruited through professional associations (e.g., 


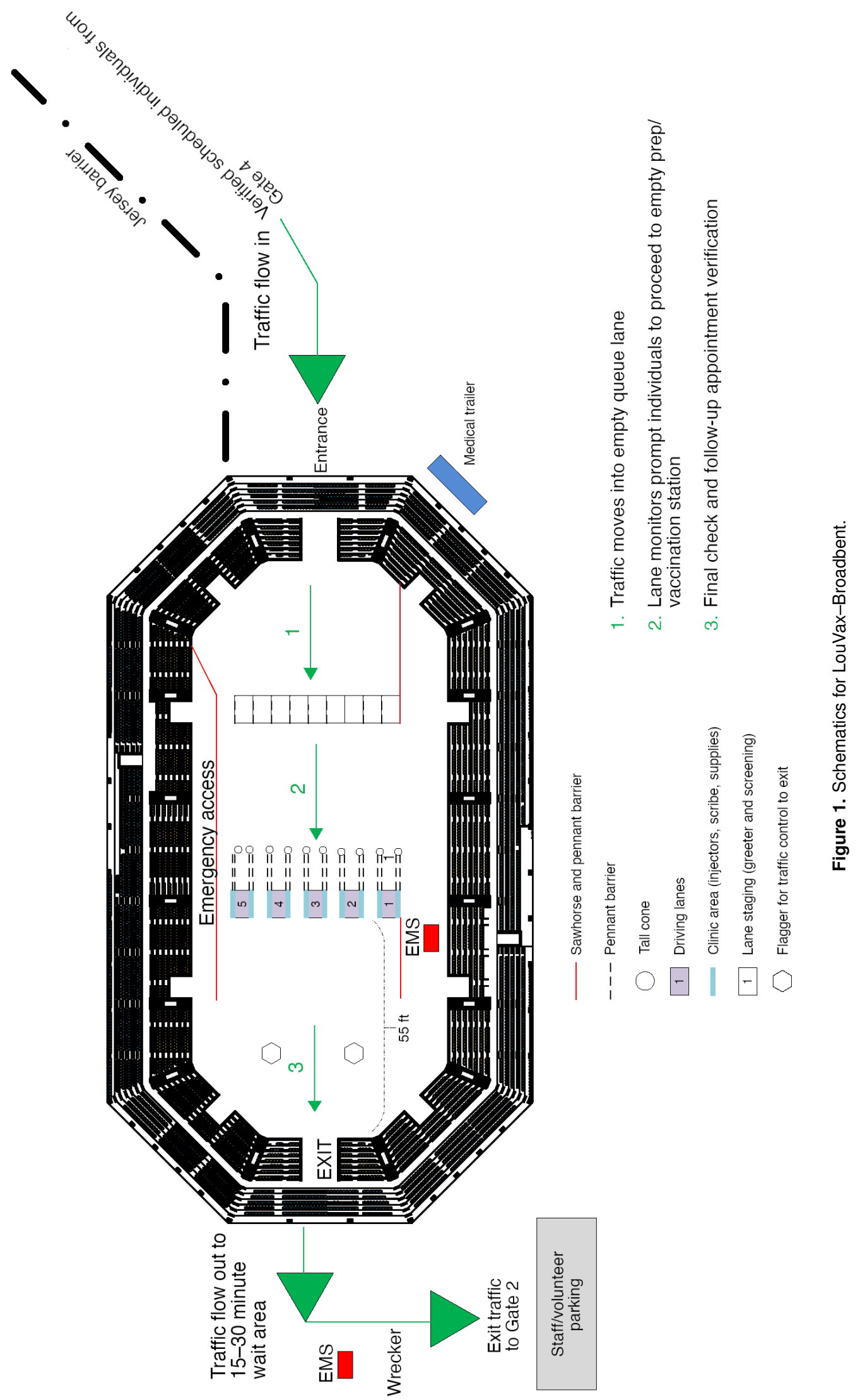




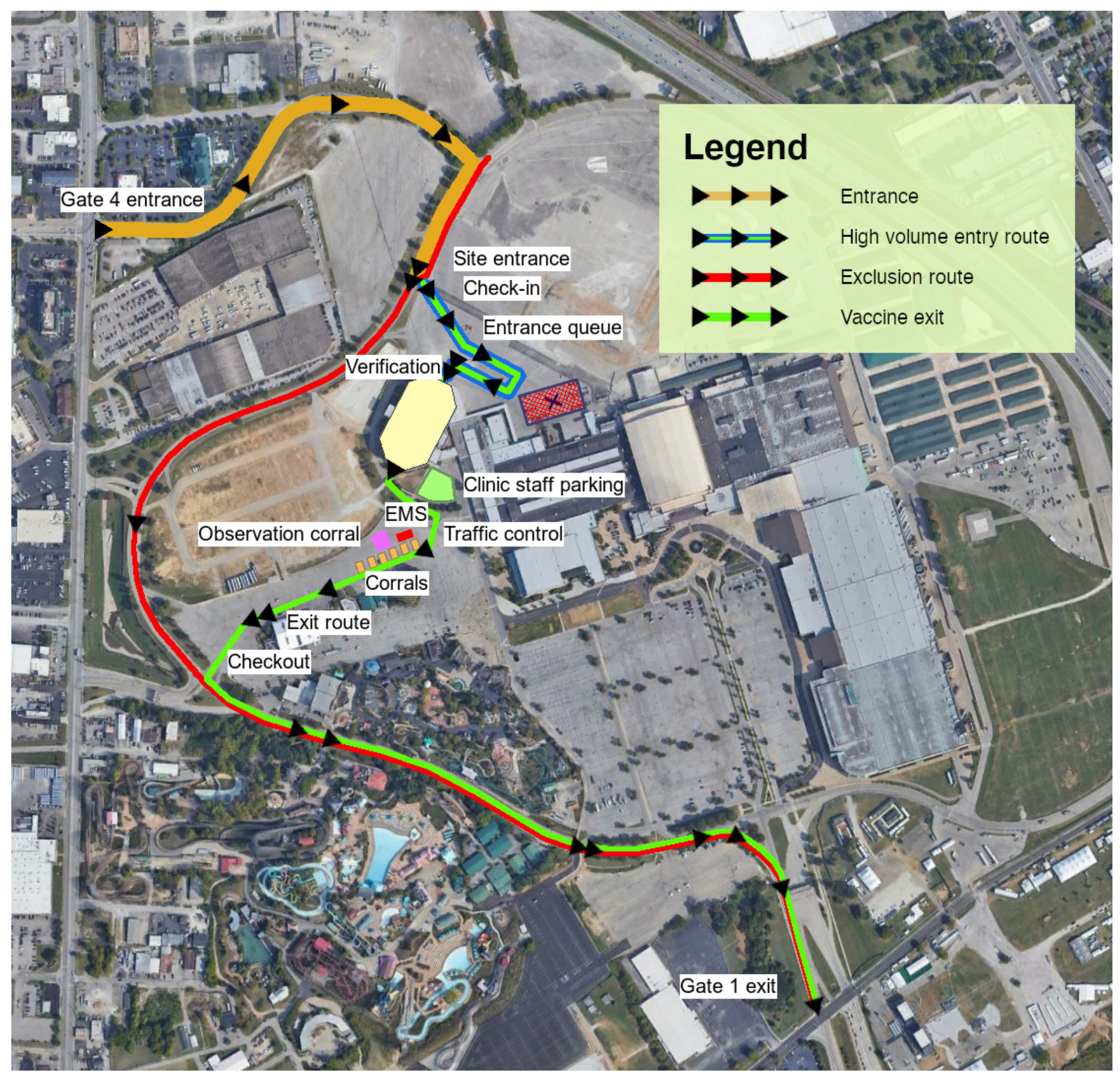

Figure 2. Aerial view of the arena and adjacent areas. 


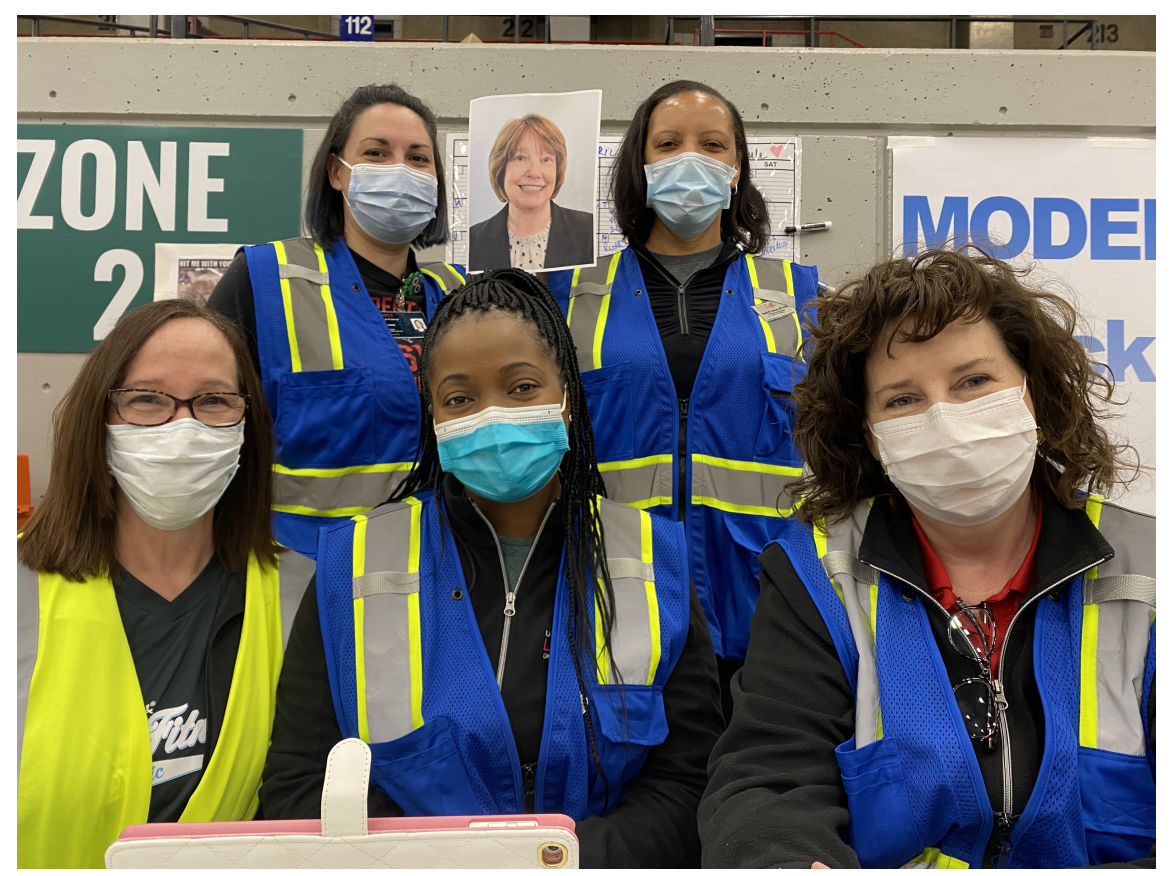

Figure 3. Blue Vest medical team.

Greater Louisville Medical Society, Kentucky Nurses Association) and the media/social media. All volunteers were eligible for vaccination after volunteering for forty hours, or often sooner. Louisville Metro Government and LMPHW personnel provided leadership and management oversight. A typical day's workforce would consist of 115 volunteers and 39 LMPHW or Louisville Metro Government employees. All volunteers were on-site, with a portion of LMPHW or Metro Government employees at off-site locations (e.g., registration call centers). Zone 3 also required an ambulance and two emergency medical technicians (EMT)/paramedics, employed by the Louisville Metro Government, on site at all times. The Incident Commander and Medical Director (physician), as well as a group of Louisville Metro Government and LMPHW employees, were on site every day as part of Command Center operations. An advanced practice registered nurse (APRN) with expertise in immunization, infection control, and education was a primary planner for Zone 2 operations and was on site daily throughout the entire vaccination event. The medical team working with this APRN planner consisted of advanced practice registered nurses and registered nurses with expertise in mass immunization, infectious diseases, infection control, adult and pediatric healthcare clinical practice, and adult education. This volunteer medical team represented CETIP at the University of Louisville and the Kentucky Nurses Association. The volunteer and paid employee workforce staffed each of the three zones of operation, with the vaccination site doors opening for operation Monday-Friday at 08:00 and closing at 17:00 or whenever the last cars were through the process. Volunteers were enrolled into a sign-up and notification system (MyImpact ${ }^{\mathrm{TM}}$ ) managed by the Louisville Metro Government. Vetting of licensure occurred as part of initial volunteer enrollment. Weekly notices were sent to the volunteer workforce via email, outlining specific areas of need within the three zones (e.g., vaccinator, pharmacy volunteer, screener). Volunteers signed up to work in a designated role for a four-hour shift (07:30-11:30, 11:00-15:00, 14:30-17:30), with overlap occurring to allow time for training, process updates, shift changes, and end-of-day procedures. Volunteers working in the pharmacy area in Zone 2 or those administering vaccine were licensed healthcare workers either currently employed or retired. The pharmacy area was also staffed by licensed healthcare workers and always included at least one licensed pharmacist. Volunteers working in all other areas represented a variety of skills, backgrounds, and age groups. All volunteers, without exception, were trained prior to beginning their shift. Aspects of training were focused on the competencies required for the roles. Training procedures, training manuals, tools, and resources for training were developed by CETIP personnel and updated frequently as part of continuous performance assessment and improvement.

The medical team was responsible for setting up Zone 2 vaccination areas, training, continuous performance assessment and improvement, addressing questions involving vaccines and the vaccination process, managing concerns regarding screening and safety, and trou- 
bleshooting. During the first seven weeks of operation, members of the medical team set up the pharmacy area for use each day. With the expansion of the pharmacy team, this was transitioned to a pharmacy team responsibility, and both pharmacy and medical teams worked together to provide orientation and training for those responsible for vaccine handling, management, and preparation. Members of the volunteer medical team wore blue vests, so they were easily and quickly recognized and identified (Figure 3). At least one "Blue Vest" medical team member, often two or three, was on-site for the entire day of operation and worked with the designated site leader for Zone 2. The site leader was an employee of the LMPHW or Louisville Metro Government and reported to the site Incident Commander. This relationship facilitated rapid communication and response between the site Incident Commander and Chief Medical Officer-both employed by Louisville Metro Government and LMPHW-and the Blue Vest medical team. The pharmacy lead also reported to the Zone 2 site leader and worked collaboratively with the Blue Vest medical team.

\section{Protecting the vaccine}

Protecting the vaccine involved management and preparation of the vaccine and all prepared doses from the time of vaccine arrival at LouVax-Broadbent to the time at which remaining vaccine was returned to LMPHW at the end of the day. Vaccine protective activities included: 1) checking in of the arriving vaccine; 2 ) monitoring the temperature and environmental conditions of the vaccine throughout the preparation and dilution (if indicated) processes, 3) selection and use of appropriate needle and syringe combinations for preparation and administration, 4) procedure to prepare vaccine so that each syringe was ready for injection, 5) labeling and delivering syringes to the vaccination table, 6) maintenance of the filled syringes through delivery to the vaccination stations and until administration to the vaccine recipient, and 7) monitoring of time between vaccine vial removal from the refrigerated environment and vaccine administration.

Checking in of the arriving vaccine: Moderna and Pizer COVID-19 vaccines were available through U.S. Food \& Drug Administration (FDA) EUA and were administered at LouVax-Broadbent. As neither contained a preservative, both had challenging handling and management requirements. All vaccine was delivered by LMPHW transport refrigerators, accompanied by data loggers for temperature monitoring. Transport refrigerators contained a single type of vaccine from a single lot. If multiple lots were delivered or more than one type of vaccine was delivered, each would arrive in a separate transport refrigerator and would remain separated throughout the vaccine event day. Hourly monitoring and written documentation of temperature, as well as monitoring of the data log- ger, was recorded and maintained as is standard practice. An on-site pharmaceutical-grade vaccine refrigerator / freezer was also made available for storage of vaccine if large numbers of doses were being prepared and additional storage was needed. This refrigerator also maintained a temperature data logger for both refrigerator and freezer compartments, and documentation of temperature was also done manually. In addition, a digital thermometer documenting the ambient temperature of LouVax-Broadbent was monitored and documented to ensure that doses of vaccine were not subjected to unfavorable temperature conditions after syringe preparation and while awaiting administration.

Vaccine delivered from LMPHW would be accepted for receipt by a pharmacy volunteer each morning at approximately 07:00. Temperature verifications during transport, lot numbers of the vaccine vials, and the total number of vials and doses delivered were verified and documented. Stickers that would be used to place on patient document forms and the Centers for Disease Control and Prevention (CDC) vaccine cards were printed at LouVax-Broadbent each morning once vaccine lot numbers and doses had been verified. Pages of stickers would be delivered to the vaccination tables along with the prepared syringes. Stickers for the Pfizer vaccine were printed with black ink on white stickers, and a purple highlighter line was drawn, thereby visually indicating the Pfizer vaccine. Moderna stickers were printed with light blue ink on white stickers. This color-coding approach was used throughout the process as an error-proofing step. Once all vaccine had been verified and checked-in, and lot numbers on printed vaccine stickers were confirmed, vials were made available to the pharmacy team to begin preparation for that day's event. Vials were removed from refrigerators and provided to the pharmacy volunteers according to the number of registered vaccine recipients. The goal was to ensure that no vaccine was prepared or outside the refrigerated environment for more than four hours before administration to an individual.

Monitoring temperature and environmental conditions of the vaccine: The Broadbent Arena environment is similar to a giant garage, so special considerations for protecting the vaccine were implemented. Three biosafety cabinets were borrowed from the University of Louisville to better provide a clean environment for preparing and filling syringes with the vaccine (Figure 4). It was determined that all vaccine would be handled in a designated and restricted area, with vaccine vials opened and vaccines prepared in the biosafety cabinet ("under the hood"). The cabinets were transported to the arena by physical plant personnel. Once in place, they were decontaminated and certified for use by an external partner using standard practices reviewed and approved by University of Louisville Department of Environmental Health and Safety personnel. Protocols were developed for open- 


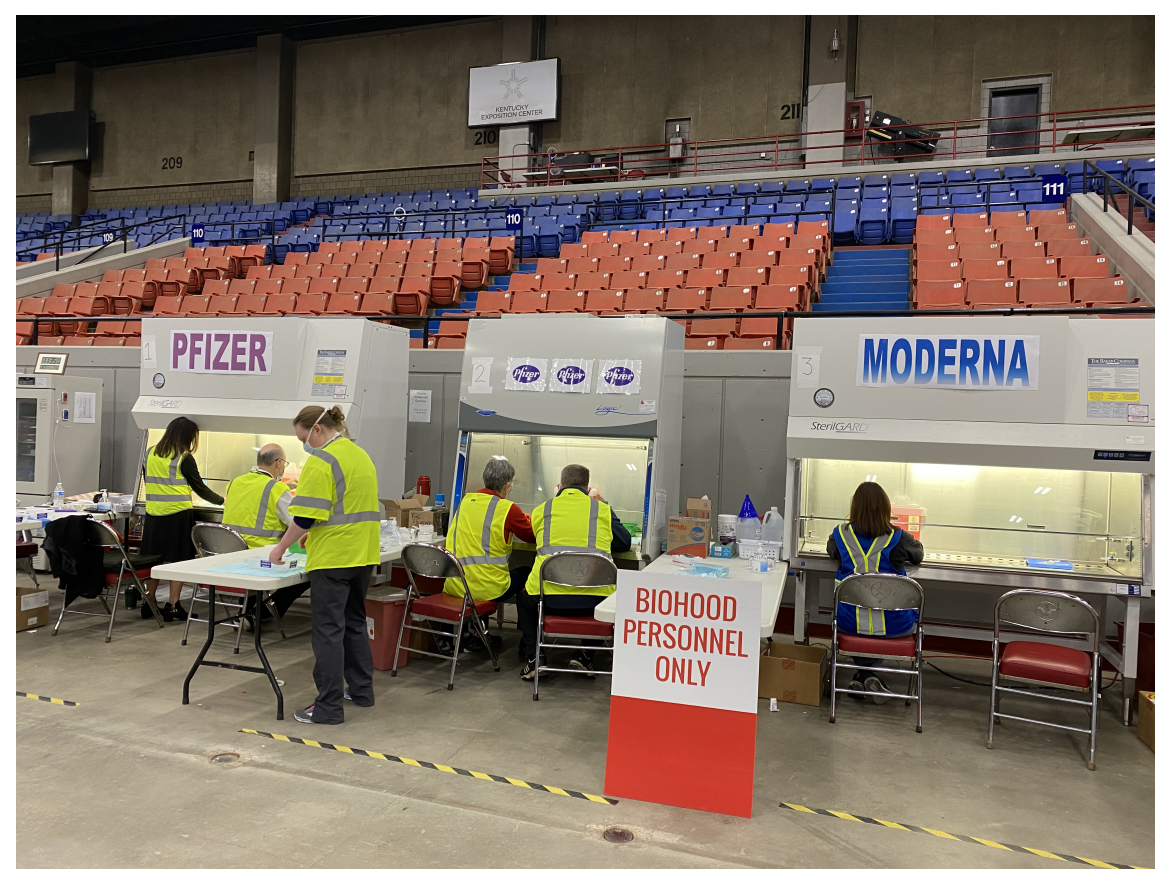

Figure 4. Pharmacy area and biosafety cabinets.

ing (beginning of the day) and closing (end of the day) of the biosafety cabinets to ensure daily cleaning and disinfection.

Selection and use of appropriate needle and syringe combinations: Personnel responsible for handling the vaccine and preparing syringes were trained by the APRN medical team planner and a practicing pharmacist to ensure competence. At least one registered pharmacist was on site every day throughout the entire LouVax-Broadbent operation, with four to six additional personnel working as part of the pharmacy team. The team also included a pharmacy lead, a vaccine accountability lead, and a runner. The entire pharmacy team consisted of at least eight individuals with demonstrated competence in aspects of vaccine handling, management, and preparation. Each day, the pharmacy lead evaluated all needle and syringe combinations in the ancillary kits provided by the individual vaccine manufacturers. This ensured adequate supply and also ensured that the combinations were appropriate for the vaccine. For example, the Pfizer vaccine required dilution, so separate syringes and needles for dilution were needed. In addition, needles and syringes with low "dead space" were essential to ensure that all doses could be completely withdrawn from the vaccine multidose vials. Evaluation of the needle and syringe supply was done the day before they would be used as a means of providing appropriate and adequate supply on site for the next vaccination day. Figure 5 shows the many needle and syringe combinations included in the ancillary kits provided by the vaccine manufactur- ers and delivered with vaccine supplies.

Withdrawing vaccine from vials, dilution, mixing, and preparing syringes for administration: Training for all pharmacy team members focused on safe injection practices, including hand hygiene, use of the biosafety cabinet, and clean and sterile techniques. Pharmacy and CDC standards were used as a basis for training. $[9,10]$ All syringes were opened and filled under the hood by members of the pharmacy team. Pfizer vaccines were handled in a biosafety cabinet labeled with a large "Pfizer" sign, and Moderna vaccines were handled in a biosafety cabinet labeled with a large "Moderna" sign. Vaccines were never combined in any biosafety cabinet as a way of preventing error. A middle biosafety cabinet was used to pre-draw the saline required for reconstitution of the Pfizer vaccine and served as a 'buffer zone' for clear separation of the two vaccines.

Differing dosages, dilution processes, and preparation logistics made this a particularly challenging step in the process. To facilitate efficiency and prevent error, the processes were divided into specific steps, with personnel assigned specific responsibilities. For example, one individual would be responsible for filling syringes used for diluting the Pfizer vaccine. Sterile syringe tips were purchased to enable that individual to fill multiple syringes in immediate succession. Syringes remained under the hood for use throughout a single volunteer's four-hour shift. Each syringe was checked for appropriate volume by another designated volunteer. 


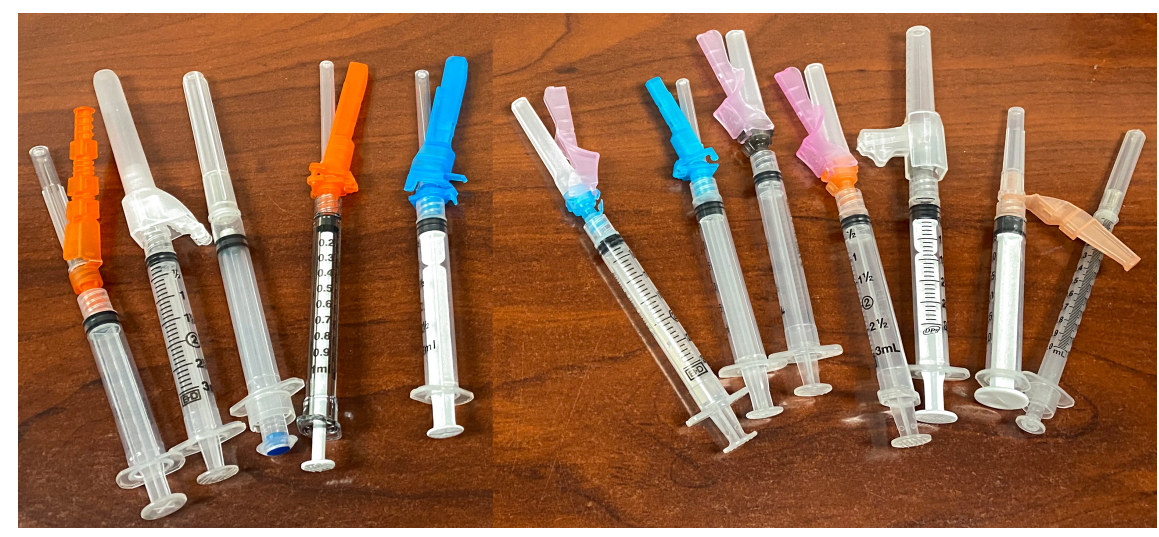

Figure 5. Needle and syringe combinations provided in ancillary supply kits.

Competencies were developed to help guide training and identify gaps in demonstrated abilities. For example, a training vial was tinted with food coloring and used to demonstrate the dilution volume as well as doses. This enabled individuals assigned to the pharmacy area to demonstrate competence while also enabling the pharmacy lead to correct practice prior to that person moving into the syringe production process.

Once the saline syringe had been prepared and checked, it was passed to another pharmacy team to dilute the vaccine and withdraw the dose amount. The diluent syringes were $3 \mathrm{cc}$ in volume with a black syringe tip, so it was easy to visually identify them. All syringes prepared for a single day were used during that day. Beginning with the last shift of each day, diluent syringes were prepared at a cadence that was more real-time to minimize waste as any diluent syringe remaining unused at the end of a vaccination day would be discarded. When vaccine (Pfizer) was ready for preparation, the pharmacy team would place a sterile needle onto the tip of the saline syringe and inject the entire amount into the undiluted vaccine vial. Diluted vials were handed to another pharmacist to withdraw into syringes prepared for administration. This process prevented errors in dilution (e.g., failure to dilute or multiple dilutions) in any given vial. Syringes would be prepared in groups corresponding to the number of doses withdrawn from that vial. For example, a vial of Pfizer vaccine would provide six doses, so all six doses would be withdrawn and placed into a single tray for dose verification. Pharmacy team members would use the syringe and needle combinations selected for that day and prepare four syringes with a 1-inch needle and two syringes with a $1 \frac{1}{2}$-inch needle to allow for intramuscular administration to patients of all body sizes. The vial would remain in the tray with those six syringes until all had been dose-checked by another pharmacy team member. If any syringe was determined to contain either more or less than the required $0.3 \mathrm{~mL}$ dose, the tray was returned to the hood so dosing in that/those syringe(s) could be adjusted. On occasion, an air bubble would be identified in a syringe, necessitating that additional volume be added to that syringe. Completed and checked trays would be placed in a designated area for labeling and documentation. The same processes were used to fill syringes with Moderna vaccine, but without dilution. Trays of 10 syringes would be prepared, six with 1-inch needles and four with $1 \frac{1}{1} 2$ inch needles, with a double-checking process for dose volume performed as was done with the previously described Pfizer doses.

Labeling vaccine doses and delivery to vaccination tables: Prepared trays of filled syringes were labeled with a sticker noting the name of the vaccine. Purple Pfizer and light blue Moderna stickers were used. Once the prepared trays of syringes had completed the quality check (e.g., ensuring correct dose, all needles and caps checked for tight connection), the trays were placed inside 1-gallon resealable plastic bags, and the time at which the vaccine vial was removed from the refrigerator was written on the outside of the bag. Personnel at the vaccination tables were instructed to maintain trays inside the bag, enabling visual checks to monitor time out of the refrigerator for each dose. When trays were empty, personnel at the vaccination table alerted the pharmacy runner, who could retrieve the tray and bag and return both to the pharmacy area. Trays were disinfected and placed back into the production line. The time on the plastic bag was crossed out and the bag reused throughout that day.

Maintenance of the filled syringes: Filled syringes remained at each vaccination table until all were used. If a syringe or needle was contaminated (e.g., dropped on the ground), the pharmacy runner was notified so that the syringe could be returned to the pharmacy area for disposal and documentation. If there was need for a syringe with a different needle size (e.g., a longer needle was needed but not available at the table at that time), 


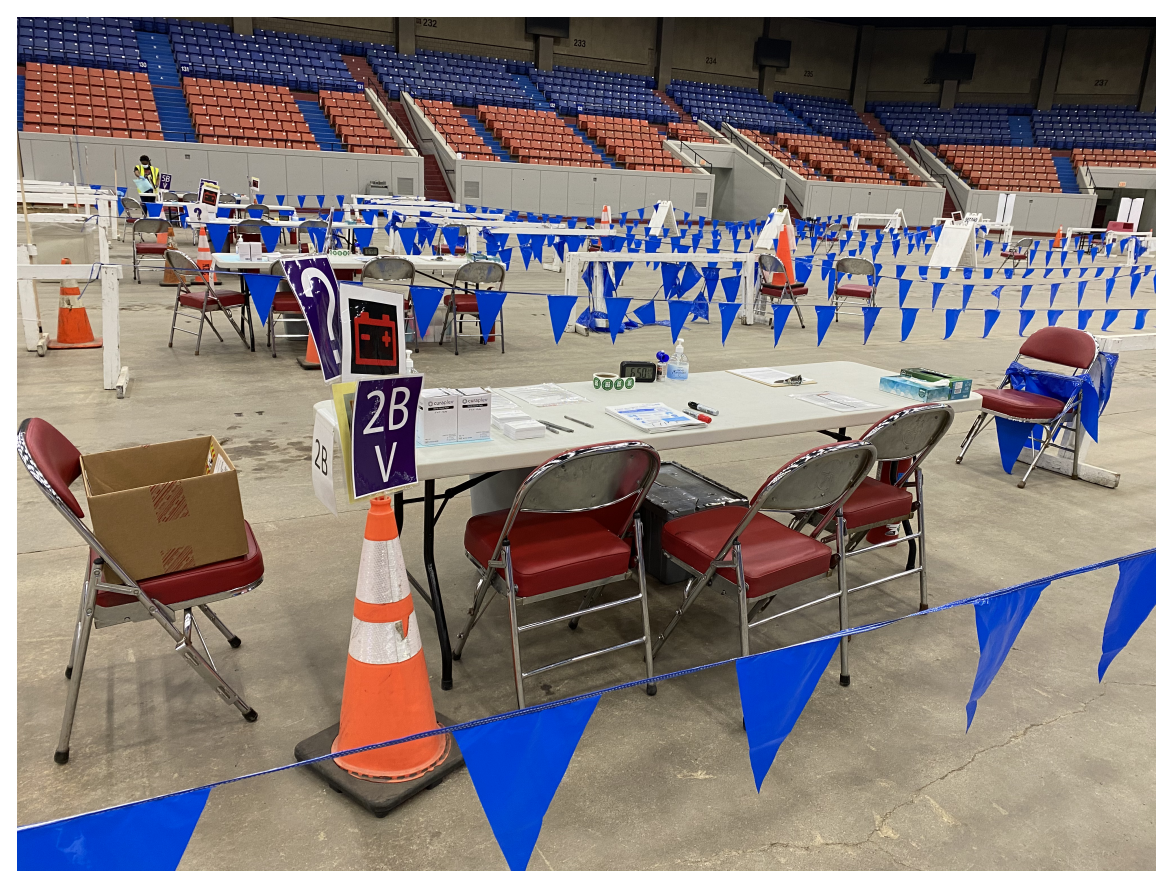

Figure 6. Signage for communication.

the vaccinating table personnel would notify the pharmacy runner, who could exchange a syringe from one vaccination station for the next or return the syringe to the pharmacy area, where a new needle could be applied under the hood. No needle changes were allowed to be done outside the pharmacy biosafety cabinet. At the end of the vaccination day, any remaining vaccine doses were returned to the pharmacy area. Those doses were used for any unvaccinated volunteer or individuals who were on a waiting-to-be-called list. If a dose was unable to be administered, it was destroyed and documented in accordance with LMPHW policy.

Monitoring of time from vaccine removal from refrigerator until administration: The pharmacy team leader maintained a log of the time a vial was removed from its refrigerated environment, all doses prepared, all doses of diluent prepared, any waste (e.g., syringe dropped or contaminated, or unused doses at the end of a vaccination event day), additional or 'bonus' doses obtained from a given vial of vaccine, and numbers delivered to the vaccination stations. This information was provided daily and made available throughout the day to the LMPHW chief medical officer. At the end of each volunteer shift, as part of shift change, all doses were evaluated for timing to ensure that no dose was outside the refrigerated environment for more than the four-hour goal. Remaining doses at or around the fourhour goal were identified and a plan for first administration was discussed with personnel at the vaccination stations.
Signs were developed and used by vaccinators to request specific types of vaccines. Pfizer tables, signs and syringe labels were purple and Moderna were light blue. In addition, specific signs were developed to communicate with others to indicate need for vaccine, patient-related questions, need for mechanical assistance with cars, or help with technology (Figure 6). This helped to visually facilitate communication between vaccinators, pharmacy runners, and support personnel, and helped the Zone 2 Blue Vest team to identify practice deviations and real-time needs at the vaccination tables while also minimizing wasted doses.

\section{Protecting the vaccine recipient}

Protecting the vaccine recipient required that the process: 1) uniformly screen all recipients prior to vaccination, 2) ensure infection prevention and control throughout the vaccination process, 3 ) ensure safe injection practice, 4) use administration techniques to facilitate intramuscular injection of the vaccine, 5) document the screening and vaccination process, 6) recognize and respond to immediate adverse events, and 7) provide patient education, including orientation to CDC V-safe, the adverse event monitoring system available via cell phone. Standardized training procedures were developed, and group training for all personnel in Zone 2 was done at every shift change, regardless of how often an individual had participated as part of LouVax-Broadbent. 


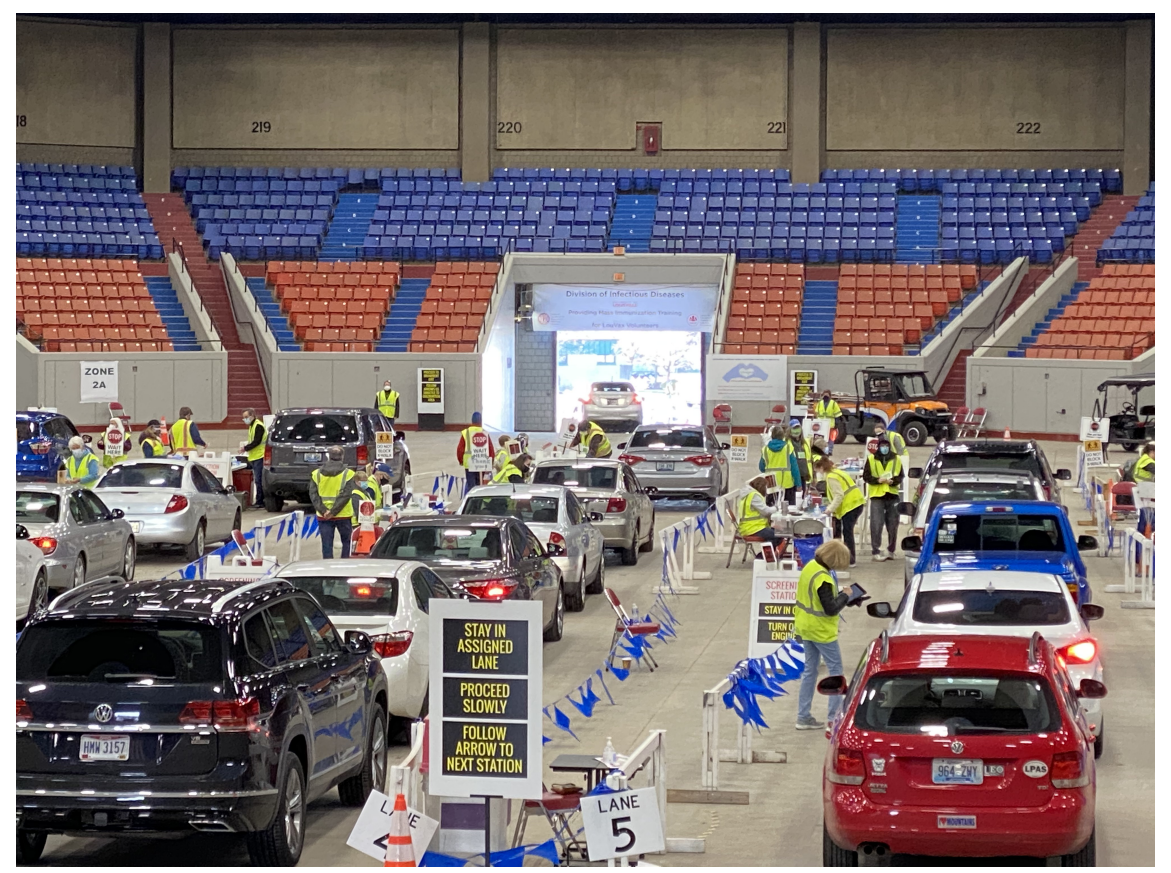

Figure 7. Inside LouVax Zone 2 in operation.

Uniform screening: LouVax-Broadbent was structured so that there were six drive-through lanes and a seventh station that provided an area for walk-up traffic as well as spaces for $4-5$ cars to stop and park, if needed. Individuals were directed to enter one of the six lanes as they entered the arena from Zone 1 outside the arena. Once inside Zone 2 and in a lane, a greeter would go to the car and begin a screening process, asking about current illness and checking temperature with an infrared scanning thermometer. If an individual registered a temperature greater than $100{ }^{\circ} \mathrm{F}$, the Zone 2 Blue Vest was alerted to provide a more in-depth assessment. Zone 2 lanes and the LouVax arena are shown in Figure 7. In the event that an individual was deemed unable to be vaccinated (e.g., ill, still in quarantine period), a card was placed on the outside of their car windshield-indicating movement through the lane without vaccination-and that person was rescheduled to return for vaccination at a later date. If special needs were identified, such as an unanticipated need for an interpreter, a sign was placed on the dashboard of the car directing them to Zone 2A, the location designated for walk-up vaccinations and special needs, where interpreter services would be provided (Figure 8). After temperature screening, vaccine recipients were moved forward in their individual lanes toward the vaccination tables.

Infection prevention and control: All personnel working inside in Zone 2 also had temperature checks prior to entering the arena area, and all were required to wear medical-grade masks. These masks were pro- vided by LMPHW and made available for all personnel at the point of entry into LouVax-Broadbent. Face coverings could be worn in addition to masks, at the preference of the volunteer, but not as a replacement for medical masks. Boxes of masks were visible and accessible throughout the site to ensure immediate availability. All personnel were provided with a dress code that included closed-toe shoes in case a needle were to be dropped. Setup and end-of-day procedures included cleaning and disinfection of all surfaces using germicidal agents included on the Environmental Protection Agency (EPA) List N.[11] Biosafety cabinets were cleaned and disinfected according to a written protocol at setup and the end of the day. All supplies available for use at the event, including ancillary kits, personal protective equipment (PPE), and clerical supplies, were stored in a supply/logistics area maintained by LMPHW personnel, the Blue Vest team, and the pharmacy lead (Figure 9). Alcohol-based hand rub was available at every vaccination station, at the pharmacy area, and at all greeter stations. Orientation for each session included a review of mask use and hand hygiene. Gloves were made available at each vaccination station. Injection technique focused on CDC guidance, Occupational Safety and Health Administration (OSHA) bloodborne pathogen standards, and infection control practice, which all stressed the use of gloves as protection against blood/body fluid exposure and not as standard injection practice equipment. Protecting the vaccine recipient through evidence-based infection prevention and control competencies was the foundation for this aspect of orientation and practice monitor- 


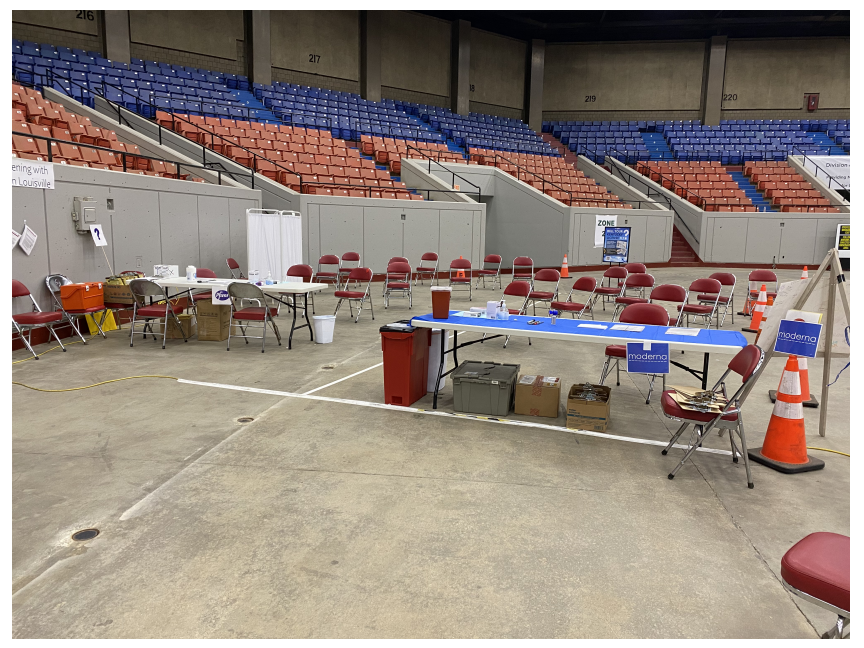

Figure 8. Zone $2 \mathrm{~A}$ for walk-ins and special needs showing colorcoded table covering.

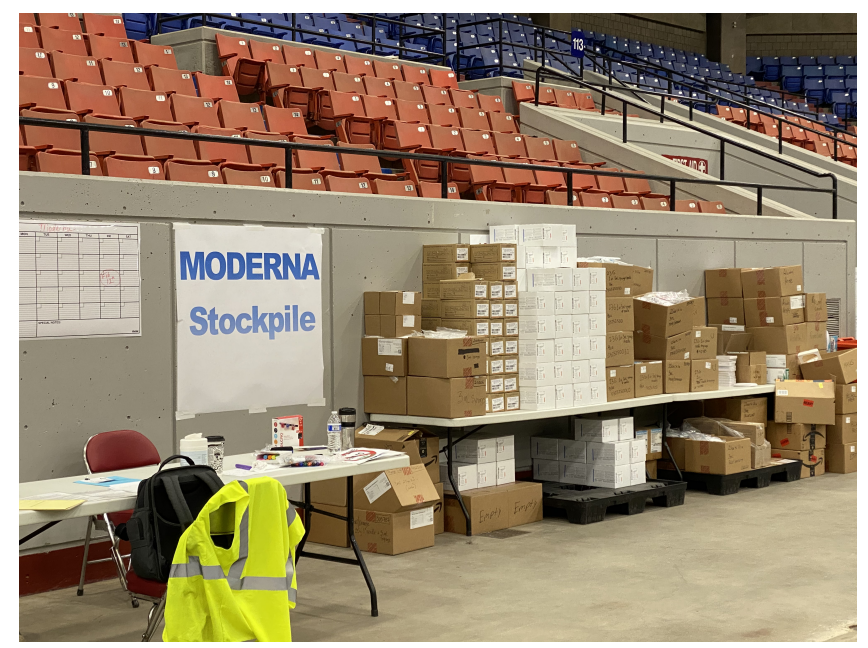

Figure 9. Supply/logistics area. ing.

Safe injection practices: Each day, syringe and needle combinations that had been provided in the vaccine ancillary kits were reviewed with volunteers. Signs indicating the "Syringe and Needle Combination of the Day" were used during orientation and remained posted throughout the session (Figure 10). If, at any point during the day, those combinations were changed (e.g., due to availability), a new sign was made and information shared with each vaccinator. The syringe and nuances that may impact administration of the entire dose were reviewed. An example involved the use of syringes with retractable needles and the need to completely inject and initiate needle retraction while still in the muscle of the recipient. Any syringe or needle contamination, or any concern about the sterility of the needle/syringe or the vaccine, prompted immediate sequestering of the item(s) and return to the pharmacy area for review, investigation, and documentation.

Administration techniques: All of the COVID-19 vaccines were to be administered intramuscularly (IM), with the deltoid muscle as the primary injection site of choice. As LouVax-Broadbent operated as a drivethrough event, individuals received their vaccine while remaining in their vehicle. The technique for deltoid injection varied with the position of the recipient, height of the vehicle, height of the injector, and preference of the recipient. Approaches for IM administration in this setting were reviewed as part of each shift orientation. In addition, the Blue Vest team regularly scanned for Band-Aid location to identify injection sites that appeared to be too close to the shoulder joint (potentially too high) and those that appeared to be too close to the lower aspect of the upper arm (potentially too low).
Technique reviews were performed regularly throughout each session. Hand hygiene practices and immediate activation of safety devices were also monitored during the walking rounds made by the Blue Vest team.

Documentation: A paper consent form was used throughout the 17-week operation of LouVaxBroadbent. Efforts were made to move to an electronic documentation process through a cloud-based application program developed in SalesForce ${ }^{\mathrm{TM}}$. Major challenges to use of the electronic system for documentation (e.g., reliable internet, ability to identify individuals in the system, ease of use, ability to quickly learn and use the system, willingness to learn and use the system, changes and enhancements to the system) existed among the volunteer workforce. Therefore, use of a paper consent process that could serve as a reliable source document continued throughout the LouVaxBroadbent operation as parallel documentation. The consent form contained demographic information, screening questions, and an area for documentation of the vaccine brand, dose, lot number, expiration date, anatomic site of administration, date of administration, and the name and credentials of the injector. Each vaccine recipient was also given a CDC vaccination card with a sticker containing the name of the vaccine, dose, date of vaccination, lot number, and site of administration (LouVax-Broadbent). The vaccine recipient name and date of birth were handwritten on the card, and a date of return for second dose was entered on the back of the card ( 21 or 28 days later). Individuals returning for a second dose of vaccine without their original CDC vaccination card would have their first dose verified by review of the Kentucky Immunization Registry (KYIR), and a new CDC vaccination card with documentation for both doses would be provided. 


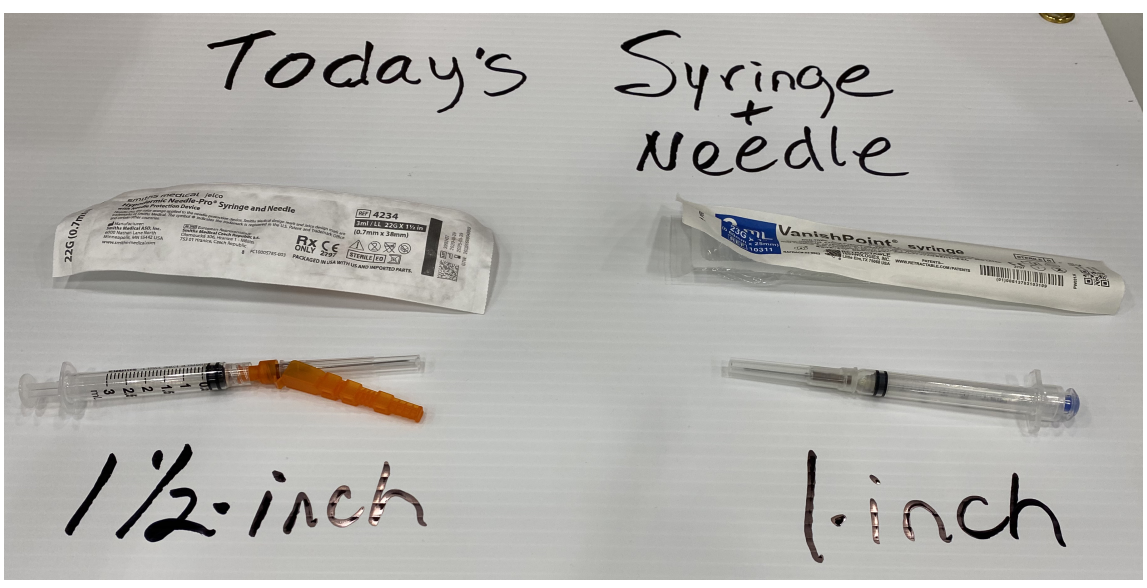

Figure 10. Needle and syringe of the day.

Adverse events: Monitoring for immediate adverse events required that all individuals at the vaccination tables be aware of clinical presentations and 'complaints of significance,' have competence in patient assessment, and have a low threshold for sign and symptom 'acceptance.' Orientation included a review of common occurrences post-vaccination, such as syncope, other immediate complaints, such as itching or respiratory concerns, and first-line intervention, including changes in positioning, removal of seat belts, and opening of car doors. An emergency drug box was readily available and included written protocols for use of oral diphenhydramine and epinephrine autoinjectors, both of which were included in the drug box. An emergency air horn was located at each vaccination table so that a call for rapid assistance could be initiated and the vaccination lines stopped until deemed appropriate to restart by the Blue Vest team leader and/or the LMPHW Medical Director. Emergency Medical Services personnel were on-site in Zone 3 for postvaccination monitoring once a car had left the Zone 2 vaccination area. In the event that any immediate adverse event was recognized, the circumstances surrounding the event and any intervention was recorded on the consent form and in Sales Force ${ }^{\mathrm{TM}}$ if the system was operational. All Blue Vest team members had experience with emergency response post-vaccination, but each member also had a review of emergency protocols as part of their orientation and competence assessment.

Patient education: Patient education began at the time of registration prior to entering the site for vaccination. LMPHW provided electronic copies of the vaccine fact sheets and information regarding the Centers for Disease Control and Prevention's (CDC) cell phone adverse event monitoring system (CDC V-safe). Once the individuals were inside the arena, they were provided with a hard copy of the vaccine fact sheet (either Pfizer or Moderna). Children under 18 years required parental consent either in writing or verbally in direct communication with the injector. ${ }^{1}$ Patient education included information about the vaccine, expected immune responses (e.g., fever, myalgias, malaise, headache), other adverse events, and how to contact LMPHW for reporting or questions. During the first ten weeks of the event, each participant was shown a QR code and asked to use their cell phone to connect to the CDC V-safe reporting system. Individuals could complete that process during their postvaccination wait time while in Zone 3 . After the first ten weeks of the event, V-safe information was provided electronically, and individuals were prompted to connect independently.

The drive-through process: Two vaccination tables were positioned in each lane approximately 30 feet apart, enabling multiple individuals to be vaccinated at the same time. At each vaccination table, there were three volunteer personnel: one licensed healthcare worker trained to administer intramuscular vaccination, one person responsible for completing the CDC vaccination card, and one person responsible for ensuring completion of the consent form and entering patient data into the SalesForce ${ }^{\mathrm{TM}}$ system via laptop. As previously mentioned, stickers were printed each day with the information about the vaccine doses to be administered that day. Stickers were placed on the consent form and the CDC vaccination card. Access to premade stickers assisted with time management, legibility, and error prevention. As additional assistance with second dosing and error prevention, Moderna vaccine

\footnotetext{
${ }^{1}$ The initial EUA for the Pfizer mRNA vaccine included individuals 16 and older. When LouVax opened to the general public for vaccination (i.e., after healthcare workers and teachers), children 16 and older came for vaccination. Anyone under the age of 18 years of age required parental consent.
} 


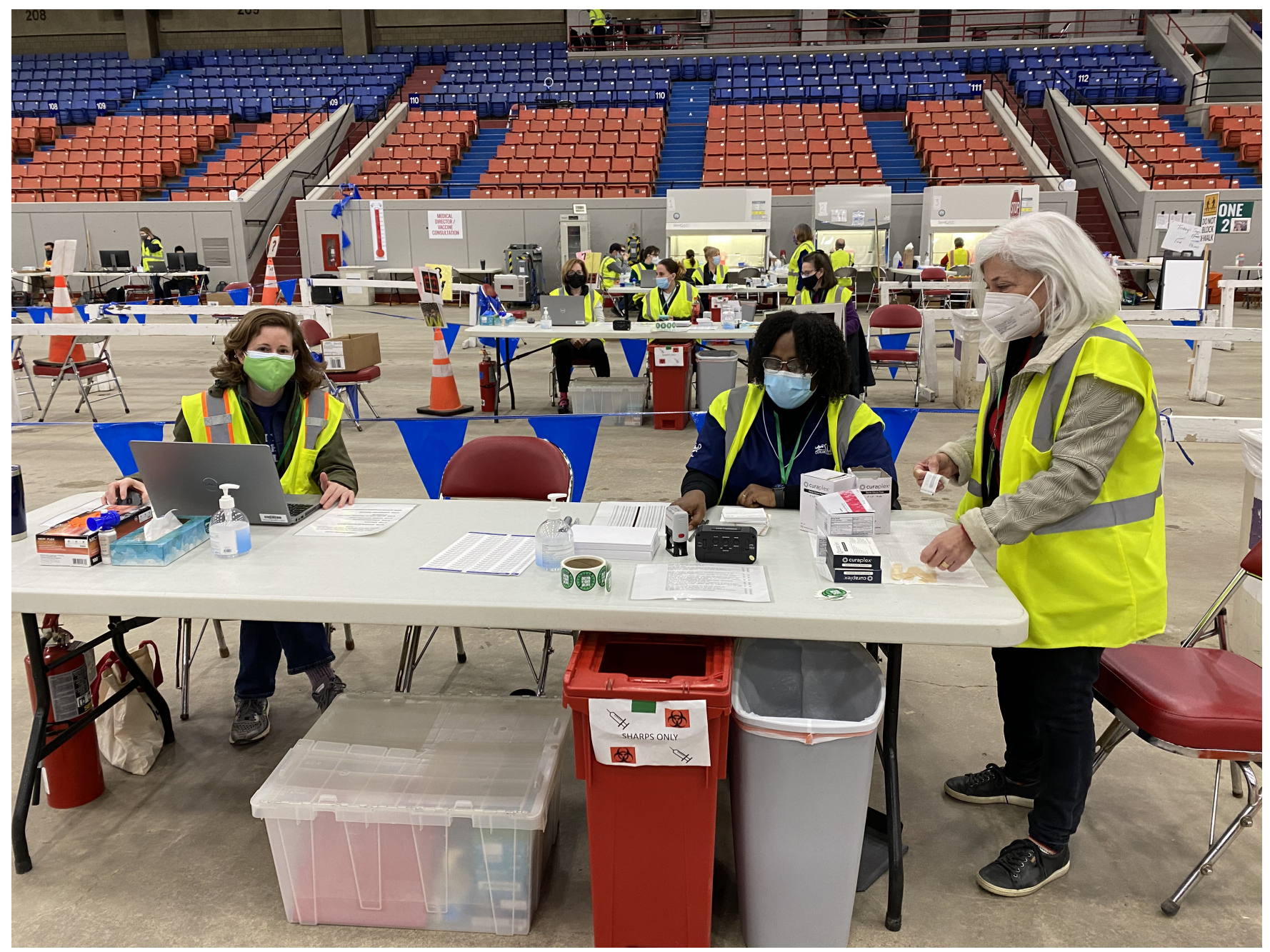

Figure 11. Vaccination stations in operation for one vaccine type.

stickers were blue print on white labels and Pfizer vaccine stickers were black print on white labels with a purple highlighter line drawn down the middle of the sticker.

During the first five weeks of the LouVax-Broadbent operation, the Moderna vaccine was the sole vaccine administered. As the Pfizer vaccine became available as part of the state's vaccine allocation, the Pfizer vaccine was added to the process. Pfizer representatives were invited to the Broadbent site, and processes and procedures for use of that vaccine were reviewed and demonstrated. Procedures for storage, handling, and dilution were written and incorporated into the training provided for all Zone 2 personnel. "Dry runs" were conducted to identify process concerns and bottlenecks, and staffing numbers and responsibilities were adjusted. This became particularly important for volunteers assigned to the pharmacy area. By this time, second-dose Moderna vaccines were beginning to be administered, so it was determined that the LouVaxBroadbent process would include access to both Pfizer and Moderna vaccines throughout each vaccination session. Vaccines were assigned to specific lanes (e.g., Lanes 1-3 Pfizer and Lanes 4-6 Moderna). Tablecloths were used to designate tables for specific vaccines (e.g., blue plastic tablecloths for Moderna), and signage was made for each table. Figure 11 shows the vaccination tables in operation, and Figure 8 shows tables with a Moderna color-coded table covering used when both vaccines (Pfizer and Moderna) were being administered. Zone 1 personnel directed individuals into specific lanes for access to a specific vaccine, and verification was done by greeters and vaccination personnel. As all vaccines were entered into KYIR within 24 hours of administration (transfered from SalesForce ${ }^{\mathrm{TM}}$ into the state registry), anyone reporting for a second dose had an initial verification of vaccination performed either by visualizing their CDC vaccination card or by verification through SalesForce ${ }^{\mathrm{TM}}$ or the immunization 
registry.

\section{Protecting the vaccine administration workforce}

Ensuring the safety of all personnel involved in vaccine administration was woven into the entire process, beginning with screening of individuals entering the LouVax-Broadbent site. Standardized training for all personnel included infection prevention and control practice, sharps safety, selection and use of personal protective equipment (PPE), continuous process monitoring, and performance improvement.

As previously discussed, all individuals entering the arena were screened for elevated temperature and symptom and exposure review (e.g., ill, cough, known COVID-19 exposure). Randomized COVID-19 antigen testing was also done for the volunteer workforce along with contact tracing in the event that an ill volunteer or employee was identified. In the event that any personnel was identified as antigen-positive, they were referred to a health department site for polymerase chain reaction (PCR) by nasopharyngeal (NP) swab testing, with results followed by the contact tracing team for the City of Louisville. Personnel entering Zone 2 were required to wear a medical mask at all times. Food and drink, other than bottled water, were prohibited at the vaccination tables to minimize mask removal. A break area was provided with tables and chairs positioned to promote and maintain social distancing. Training included review of infection control basics such as hand hygiene; safe injection practices; syringe and needle review, emphasizing safety devices; PPE, including mask and glove availability; and any process updates, such as lane and vaccine designations for that particular day or shift. Traffic flow for the vaccination lanes was constructed with barricades and signage. Training for all personnel included adherence to walkway instructions, location of fire extinguishers, emergency exits, and emergency exit procedures. Newsletters were developed and distributed that included changes or updates relevant to the drive-through processes and vaccination procedures. Blue Vest team members included worker safety elements in continuous monitoring and intervened in real time, as appropriate.

\section{Results}

Between January 4, 2021, and April 28, 2021, LouVaxBroadbent was open for operation for 82 days. The site was closed for 3 days due to severe weather in the Louisville area and opened on one Saturday to compensate for the closures. The average time spent on site for those receiving the vaccine was 35 minutes, including all activities from arrival through completion of the observation period. An average of 1,325 individuals were vaccinated each day, with a maximum of 1,829 vaccinated in a single day. A total of
109,529 doses of COVID-19 vaccine were administered at LouVax-Broadbent. There were no identified anaphylaxis events. Oral diphenhydramine was administered to ten vaccine recipients complaining of itching following vaccine administration.

A total of 154 personnel were needed per shift (three shifts per ten-hour day), of which 39 were paid staff (LMPHW or City of Louisville) and 115 were volunteers $(74.7 \%)$. More than 240 orientation sessions were conducted and 27 training documents developed. A total of 2,561 volunteers served the community at LouVax-Broadbent, yielding approximately $81,200 \mathrm{vol}-$ unteer hours, generating an estimated economic impact of \$2.2 million. The average volunteer served more than 32 hours, and the volunteer workforce consisted of 278 retired and actively employed registered nurses, advanced practice registered nurses, licensed practical nurses, certified nursing assistants, and medical assistants. In addition, 138 physicians, 5 dentists, 16 first responders, and 67 pharmacists provided volunteer hours. Additional volunteers represented the full spectrum of the community, including individuals with expertise in information technology, general business, and academia. Medical, nursing, and pharmacy students from six schools participated. A total of 1,134 volunteers $(45 \%)$ received their COVID-19 vaccination at LouVax-Broadbent. There were no known COVID-19 exposure events (unmasked personnel and unmasked vaccine recipient) at LouVax-Broadbent. There were three needlestick injuries reported, all occurring during activation of the safety device and none involving use of the syringes with retractable needles. Exposure protocols were immediately implemented, and there have been no identified bloodborne pathogen transmission events.

Procedures that served to protect the vaccine resulted in a waste of $132(0.12 \%)$ of the 109,529 prepared doses. Eighty-one of those 132 wasted doses occurred during the final three weeks of the drive-through event, when vaccine had become broadly available through other sites (e.g., workplace campaigns, pharmacies). Efforts to minimize waste included the use of call lists to identify those who were willing and able to immediately report to the arena for any remaining doses at the end of each vaccination day.

Keeping the flow of traffic through the drive-through process was important, so preparations were made to assist cars that had difficulty restarting following their time spent at the vaccination table. Cars were turned off while awaiting vaccination to protect the safety of the healthcare workers and to minimize engine idle time inside the arena. During the 82 vaccination days, more than 250 cars required assistance (e.g., battery jump start), with one needing to be towed. There were no vehicular accidents and no personnel injuries involving vehicles. 


\section{Lessons learned}

The 17 weeks of LouVax provided a number of important experiences and lessons learned, many focused on education and training needs to ensure competence of the healthcare workforce.

(1) Healthcare personnel, retired and actively working, were willing to participate in the drive-through immunization event as volunteers. The Kentucky Nurses Association was an important collaborator with the LMPHW in sharing information regarding the need for nurse volunteers. The Greater Louisville Medical Society, the Louisville Dental Society, and local schools of Nursing and Pharmacy were also key partners. Access to vaccine was an incentive for volunteer recruitment, as all volunteers were eligible for vaccination following completion of forty hours of volunteer service at LouVax. Often, access to vaccine occurred prior to completion of those forty hours as any doses that had been prepared for administration and remained unused at the end of the vaccination day were made available to unvaccinated volunteers. Available doses were prioritized based upon hours of volunteer service and disclosed risk factors of the individual volunteers seeking vaccination (e.g., age, underlying health conditions). Interestingly, the vast majority of volunteers continued to volunteer at LouVax after receiving vaccine.

(2) Nursing student groups were regular participants in Zone 2. For students to be engaged in vaccination, they were required to have their instructor present and responsible for oversight. All students and instructors were required to participate in the orientation training. Location of student groups was an important consideration for the Blue Vest team as the students required additional time for vaccination. Therefore, placement of student groups varied according to the number of volunteers, anticipated numbers of individuals coming to the site for vaccination, scheduled walk-in groups, and the length of time for which the students were able to participate (e.g., any limitations in clinical hours).

(3) A web-based platform to enable volunteer signup and active two-way communication was critical in recruiting volunteers as well as organizing daily activities. The Blue Vest team reviewed volunteer lists daily and used that information to allocate staffing for the vaccination tables.

(4) Regular changes in supplies and equipment available for use by the volunteers required daily review and education. Ancillary supply kits were available at the arena, and supplies were prepared for the next day's use. Key to this preparation was identifying the needle/syringe combinations to be used the next day. This enabled training for pharmacy personnel and preparation for training of the next day's volunteers.
(5) Provision of standardized training and participation in training prior to volunteering for a given shift enabled consistent performance among the volunteers with respect to infection control and personnel safety practices. Of particular importance was demonstration of the daily syringe and needle combinations, with emphasis on safety device activation as some of those devices were not familiar to the volunteer workforce.

(6) Written training manuals available at each vaccination table enabled volunteers to have reference materials readily available. Training manuals included environmental disinfection procedures, injection procedures, patient education materials, CDC V-safe information, and post-injection adverse events and management, as well as copies of patient and provider fact sheets, consent forms with rationale for screening questions, parental consent forms, and Health Insurance Portability and Accountability Act (HIPAA) information sheets.

(7) Appropriate use of gloves and appropriate hand hygiene practice were two areas of continuous training. Some nurses were insistent upon the wearing of gloves for performance of injection. Such glove use often replaced performance of hand hygiene between patient contact and was a focus of intervention and training by the Blue Vest team. Glove use was not considered to be part of routine injection practice, but hand hygiene was. Gloves were readily available if there was concern about exposure to blood/body fluids. Injection technique reviewed at every orientation stressed approaches designed to minimize exposure situations (e.g., assisting patient with relaxation, removal of tight sleeves, positioning of the arm, use of $2 \times 2$ immediately upon removal of the needle, immediate site pressure following injection, use of Band-Aids). Some nurses stated that they regularly cleaned their gloves with alcohol hand rub as part of their standard practice. This was discouraged and, if that practice was noted, individuals were removed from the vaccination area for retraining and discussion.

(8) Clerical roles that involved documentation of information on the CDC vaccination cards and consent forms and data entry into Sales Force ${ }^{\mathrm{TM}}$ relied upon volunteers with and without healthcare experience. A common comment from retired healthcare workers involved hesitance to learn and use "another computer system." The Blue Vest team actively recruited nonclinical volunteers to perform those clerical roles and be involved in training regarding those roles. This was a critical step as it engaged an additional aspect of local community support while also removing a stressor for the volunteer healthcare workforce.

(9) Having vaccination tables disinfected and set up prior to the start of the day supported elements of training, including supplies to be used during injection, hand hygiene products, emergency air horns, training 
manuals, and vaccine stickers for the day. The Blue Vest team prepared tables prior to the opening of the site each day and worked with the last volunteer shift to remove supplies and disinfect tables at the end of the day. Twice a week, a tub of supplies was delivered to each vaccination table by LMPHW personnel. This enabled availability of standardized supplies necessary for vaccination. It also enabled evaluation of supply use in the event that information needed to be added to orientation sessions (e.g., hand hygiene supplies scantily used).

(10) Preparation of vaccine occurred in a designated pharmacy area. The three biosafety cabinets provided a clean environment. Traffic/interruption to that area was actively discouraged. Not all healthcare personnel were able to demonstrate competence in the steps necessary to withdraw correct vaccine doses into syringes or to demonstrate the steps necessary to safely and correctly dilute vaccine. This became a major area of training and competence demonstration. It became evident that approaches currently used in healthcare settings involved preparation of medication doses by pharmacy personnel. Consequently, physicians and nurses often struggled with filling syringes with correct volumes and using evidence-based infection control techniques.
Identifying healthcare workers with these competencies was one of the biggest challenges and required constant monitoring and oversight. A pharmacy lead was quickly identified and was responsible for training and practice monitoring.

(11) Debriefing sessions among the Blue Vest team, pharmacy lead, and LMPHW personnel occurred regularly as a way of identifying and sharing challenges, barriers, successes, and general information.

This mass immunization event demonstrated the depth of interest and willingness of healthcare workers and various members of the community to participate in vaccination efforts. The sense of unity against the common threat of COVID-19 provided opportunities to share and learn skills and showed a sense of volunteerism that has not been previously seen. The question now is how to continue to use this level of willingness and interest as part of the ongoing battle with this pandemic and other such threats in the future. Sharing the experiences gained through LouVax-Broadbent may help other communities to develop and implement similar approaches, including mobile vaccination events, and be confident in training approaches.
Acknowledgements: The authors thank the employees of the Louisville Metro Public Health and Wellness and City of Louisville for their partnership in this initiative. In addition, the authors thank the thousands of volunteers who worked together with a united goal of protecting their community. The authors also thank the community members who came to the drive-through for vaccination and showed their appreciation and support for the LouVax-Broadbent team.

Received: November 24, 2021

Accepted: February 8, 2022

Published: February 10, 2022

Copyright: (c) 2022 The author(s). This original article is brought to you for free and open access by ThinkIR: The University of Louisville's Institutional Repository. For more information, please contact thinkir@louisville.edu. This article is distributed under the terms of the Creative Commons Attribution 4.0 International License (CC BY 4.0), which permits unrestricted use, distribution, and reproduction in any medium, provided the original author and source are credited.

Funding Source: There was no specific funding provided for the volunteers serving this vaccination effort. Federal funding supported costs associated with use of the arena, public health, and city personnel, as well as equipment and supplies needed for the event.

Conflict of Interest: All authors declared no conflict of interest in relation to the main objective of this work.

\section{References}

1. It wouldn't be the first time you killed a bug with your car. The Courier-Journal. 1997 October 29;Advertisement. 15.

2. Carrico RM. Drive-Thru Flu Shots: A Model for Mass Immunization. Louisville, KY: Chicago Press, 2002.

3. Ungar L. Was louisville's swine flu clinic a record breaker? The Courier-Journal. 200923 November;News.

4. Carrico R, Balcom D, Buckner K, Didelot L, Garrett JH, Sullivan D. Planning and Implementing a Drive-Through Vaccination Process: A Complete How-To Manual. Louisville, KY: International Respiratory Infections Society, 2020.
5. Centers for Disease Control and Prevention. Interim guidance for routine and influenza immunization services during the COVID-19 pandemic. Available at: https://www.cdc. gov/vaccines/pandemic-guidance/index.html. Accessed 30 November 2021

6. U.S. Food \& Drug Administration. Fact sheet for healthcare providers administering vaccine (vaccination providers): Emergency use authorization (EUA) of the moderna COVID19 vaccine to prevent coronavirus disease 2019 (COVID-19). Available at: https://www.fda.gov/media/144637/download. Accessed 30 November 2021. 
7. U.S. Food \& Drug Administration. Fact sheet for healthcare providers administering vaccine (vaccination providers): Emergency use authorization (EUA) of the Pfizer-BioNTech COVID-19 vaccine to prevent coronavirus disease 2019 (COVID-19). Available at: https://www.fda.gov/media/ 144413/download. Accessed 30 November 2021.

8. U.S. Food \& Drug Administration. Fact sheet for healthcare providers administering vaccine (vaccination providers): Emergency use authorization (EUA) of the Janssen COVID19 vaccine to prevent coronavirus disease 2019 (COVID-19). Available at: https://www.fda.gov/media/146304/download. Accessed 30 November 2021.

9. Healthcare Infection Control Practices Advisory Commit- tee (HICPAC). Core infection prevention and control practices for safe healthcare delivery in all settings -recommendations of the HICPAC. Available at: https://www.cdc.gov/hicpac/ recommendations/core-practices.html. Accessed 30 November 2021.

10. United States Pharmacopeia. COVID-19 vaccine handling toolkit: Operational considerations for healthcare practitioners. Available at: https://www.usp.org/covid-19/vaccinehandling-toolkit. Accessed 30 November 2021.

11. United States Environmental Protection Agency. About list N: Distinfectants for coronavirus (COVID-19). Available at: https://www.epa.gov/coronavirus/about-list-n-disinfectantscoronavirus-covid-19-0. Accessed 30 November 2021. 